\title{
On Fate and Flexibility in Stomatal Development
}

\author{
D.L. WengieR ${ }^{1}$ AND D.C. BergmanN ${ }^{1,2}$ \\ ${ }^{1}$ Howard Hughes Medical Institute and \\ ${ }^{2}$ Department of Biology, Stanford University, Stanford, California 94305 \\ Correspondence: dbergmann@stanford.edu
}

\begin{abstract}
In plants, the development of the epidermis, and the specialized stomatal lineage within it, exemplifies an old developmental problem that is newly relevant in this current era of stem cell biology: How can a tissue maintain flexibility and change its development midcourse yet still reliably generate differentiated and patterned cells? In this perspective, we endeavor to create a conceptual framework for the widespread questions in development that are raised by observations of stomatal development pathways in "default" settings and in response to environmental challenges. These general issues are related to the molecular pathways and networks recently elucidated for Arabidopsis stomatal development. Finally, the utility of developmental approaches for solving problems of signaling specificity are explored, emphasizing the specific use of the stomatal lineage as an in vivo testing ground for hormone and mitogen-activated protein kinase (MAPK) signaling cascades.
\end{abstract}

In the developmental progression from a single egg to a complex, functional adult, several universal problems must be solved. Proliferative cell division must be balanced with terminal fate specification, cells must be organized into tissues, and the tissues of an organ connected and functionally integrated. Two major forms of life, plants and animals, generate exquisite complexity and functionality but have done so in very different ways. The particular ontogeny of plant development is, in some ways, relatively simplified in that the cell walls preclude mechanisms such as cell migration from contributing to morphogenesis and pattern. Yet, it would be naïve to consider plant development a simple process.

Owning to their presence on the plant surface, experimental accessibility, and simplified representation of major developmental themes, stomata (pores that mediate plant-atmosphere gas exchange), and the lineage from which their flanking guard cells are created, have become an excellent model for plant cell and developmental biology. The diverse patterns of stomata found on the surfaces of leaves have captured the interests of mathematicians, physicists, physiologists, and developmental biologists for many decades (see, e.g., Bünning 1953). More recently, the molecular underpinnings of stomatal development and behavior have been elucidated. In this review, the stomatal lineage also serves as an organizing concept with which to consider some core issues in plant development: How do cells acquire distinct and stable identities? How is pattern generated? How do developmental systems enable environmental modulation yet reliably generate fate and patterns? We describe specific developmental processes highlighted by stomata and then consider these processes in relation to other developmental situations in plants or animals. We find that stomata can display simplified versions of common problems but also may reveal new capabilities of plant sys- tems. After providing an updated molecular genetic inventory of the key regulators of stomatal fate and pattern, we focus on one example of a decision-making process that integrates inter- and intracellular mitogen-activated protein kinase (MAPK)-mediated signaling with a specific transcriptional output. We examine this developmental and molecular connection from two directions, exploring how development might demand certain network structures and simultaneously showing how stomata can be used as an in vivo assay for identifying specificity factors in developmental MAPK signaling.

\section{PERSPECTIVES}

\section{Cell-Fate Decisions in Plants}

A hallmark of plants is their remarkable ability to change their morphology and physiology to be in tune with the prevailing environment. The development of major plant organs, roots, shoots, and reproductive structures is not only subject to endogenous developmental programs, but these programs are guided by external information (Fig. 1). For example, many plant families modulate stomatal densities in response to climate change (Casson and Gray 2008). The leaves in which stomata reside also show environmental responsiveness. This is seen most dramatically among heterophyllics such as the Ranunculus species, with thread-like underwater leaves, and lobed floater leaves, and in the Brassica Neobeckia aquatica, whose dimorphic leaves change morphology depending on light quality (Bharathan et al. 2002). Underground, the situation repeats itself with "species-specific" root system morphologies being modulated by nutrient and moisture availability. The developmental manifestations of this environmental sensitivity present interesting problems at both logical and molecular levels. 
A
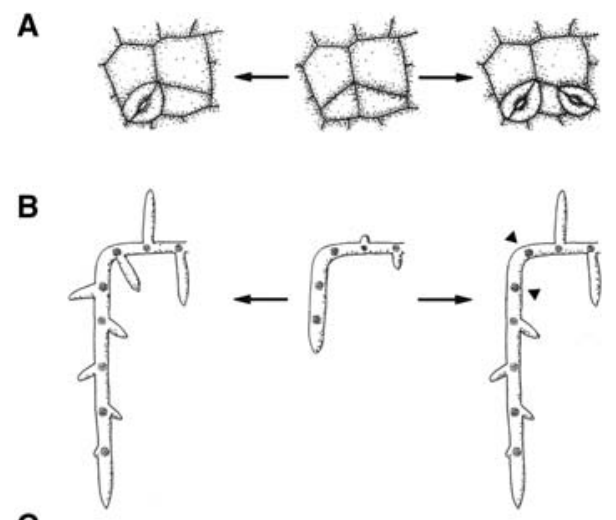

C

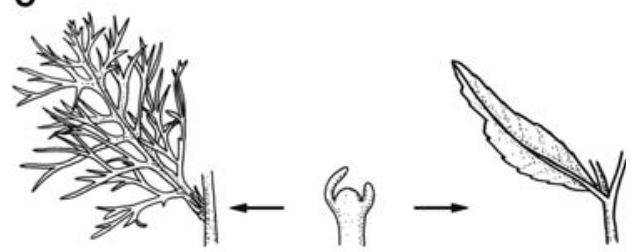

Figure 1. Developmental plasticity in response to environmental influence. From an initial primordial state (center image in each row), stomatal lineages $(A)$, roots $(B)$, or leaves $(C)$ interpret environmental cues to generate differently elaborated final forms. Arrowheads in $B$ indicate unused branch sites.

Just how flexible can plant development be? When does a cell know if should commit to a stable fate for the rest of its life and, mechanistically, how does it do so?

\section{Genetic Regulation of Stomatal Cell Fate}

To explore the issue of fate and flexibility, we describe the events and fate regulators for stomatal development and compare these with the situations in roots and leaves. In aerial organs, stomata are found on many surfaces, in species-specific patterns. In Arabidopsis, stomata are present on both adaxial and abaxial leaf surfaces (although at different densities), on stems, and on some floral organs. Stomatal pattern is subject to organ-specific rules (Berger et al. 1998; Shpak et al. 2005; Abrash and Bergmann 2010), but for simplicity, we describe stomatal development on the leaf, because this organ is where most of the core genetic regulators have been characterized. Stomata are found at both proximal and distal parts of the leaf and there is no evidence for alignment along leaf axes. Patterning is adequately described by a simple "one-cellspacing" rule that specifies that two mature stomata (each consisting of a guard-cell pair and pore between them) must always be separated by at least one nonstomatal cell (Nadeau and Sack 2002).

Stomatal fate and pattern arise from a series of precursor stages, each tied to the activity of basic helix-loop-helix (bHLH) and myeloblastosis (MYB) transcription factors (Fig. 2). Entry into the stomatal lineage correlates with stable expression of the bHLH factor SPEECHLESS (SPCH) in a subpopulation of protodermal cells (MacAlister et al. 2007; Pillitteri et al. 2007). These meristemoid mother cells (MMCs) divide asymmetrically to produce meristemoids and stomatal lineage ground cells (SLGCs), each of which, through its own developmental trajectory, ultimately produces stomatal guard cells and/or pavement cells (Fig. 3). These trajectories are interesting because they provide developmental flexibility at the cost of potential development "errors" that require extensive cellcell communication to resolve (Fig. 4). Meristemoids can undergo additional SPCH-dependent asymmetric cell divisions to renew themselves and produce sister SLGCs before differentiating into guard mother cells (GMCs),

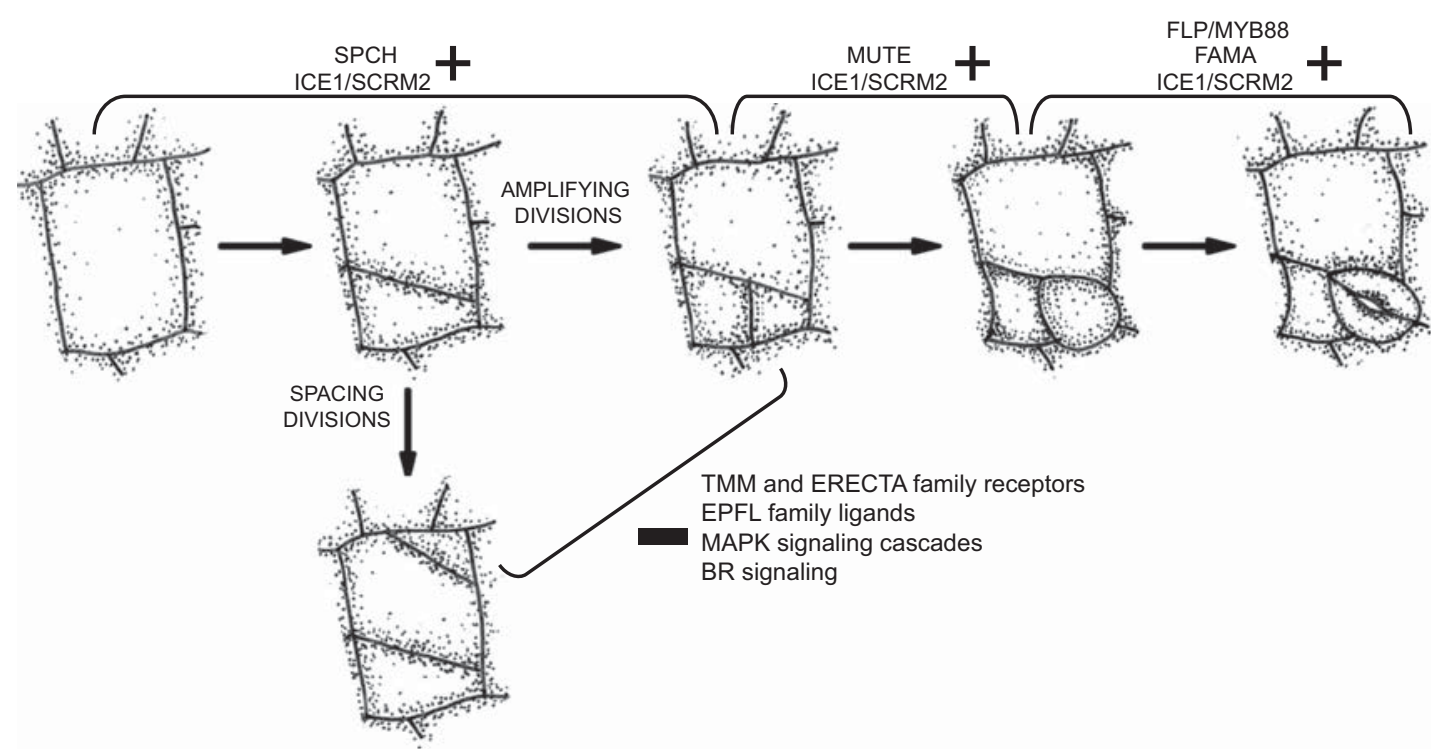

Figure 2. Scheme of stomatal development with place of action of key regulators, described in this chapter, noted. The progression of cells from early proliferative stages toward differentiation proceeds from left to right. $(+)$ Corresponds to promotion $(-)$, corresponds to repression of bracketed steps by the gene products noted. The leftmost cell (MMC) divides to create a larger SLGC and smaller meristemoid (upper and lower cells, respectively). Guard cells (bottom of rightmost complex) are preceded by GMCs. 

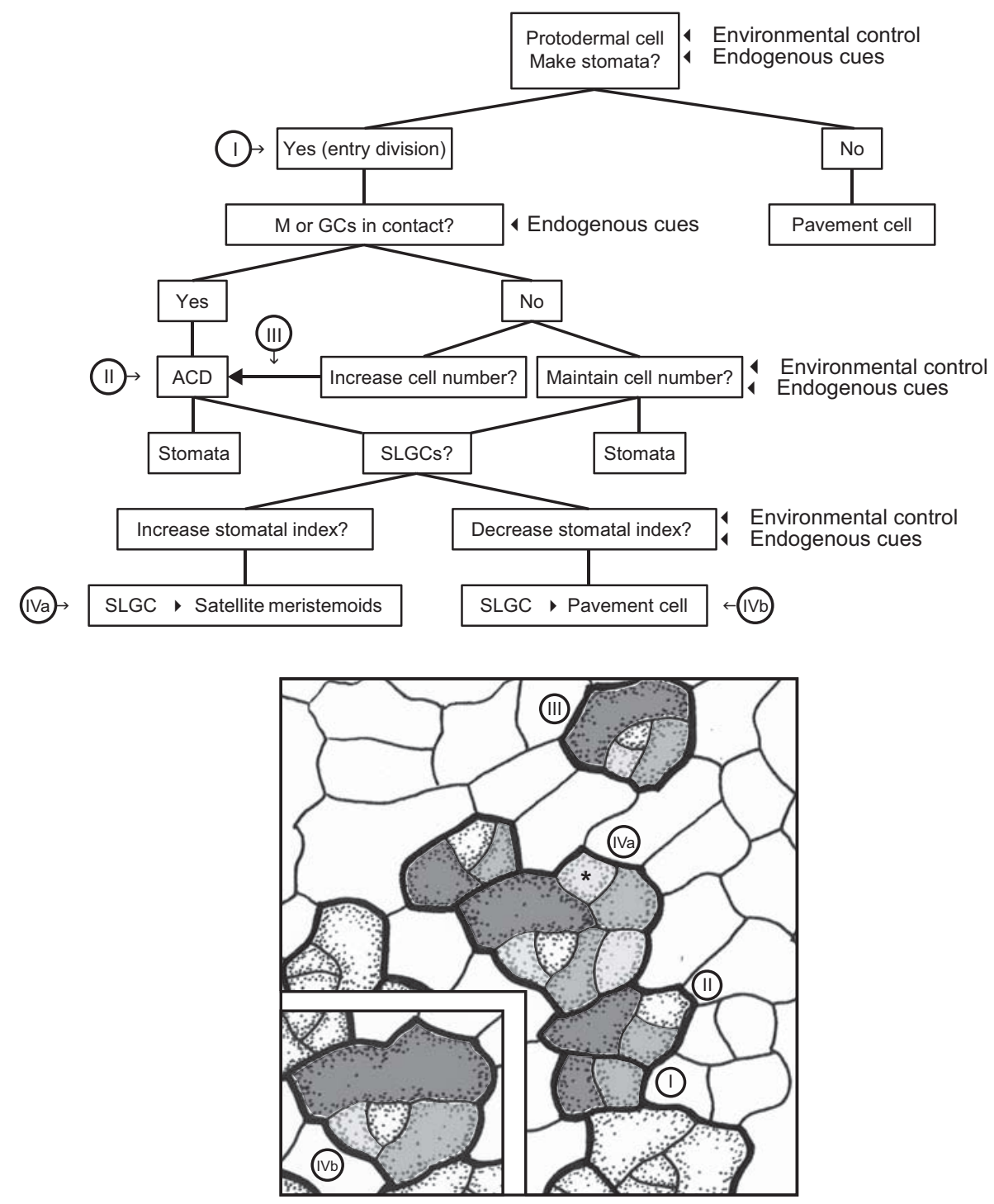

Figure 3. Decision tree for an individual stomatal precursor. In the dispersed developmental system of the leaf epidermis, the fate of an individual cell will rely on intrinsic cues ( polarity cues that ensure differences among sisters), local cues based on the state of the tissue and the cell's immediate neighbors, and global cues from distant parts of the plant and the environment. (Bottom) Based on an actual leaf, precursor cells i-iv (although present at the same time) experience different relationships with their surroundings and will likely embark on different developmental trajectories.

the immediate precursors to guard cells, whereas SLGCs can differentiate into pavement cells or undergo spatially constrained asymmetric divisions (spacing divisions) to produce new meristemoids distal to existing meristemoids, GMCs, or guard cells (MacAlister et al. 2007; Robinson et al. 2011). Expression of the paralogous bHLH (MUTE) in meristemoids (regardless of the meristemoids' origin) is required for the transition from selfrenewing divisions to the acquisition of GMC identity (MacAlister et al. 2007; Pillitteri et al. 2007). A single symmetric division of the GMC precedes terminal differentiation of its daughters, the guard cells. This cell-fate transition requires a third paralogous bHLH, FAMA (Ohashi-Ito and Bergmann 2006), and precise control over cell division also requires the R2R3 MYB trans- cription factors FOUR LIPS and MYB88; these MYBs have been shown to repress, through direct binding to their promoters, core cell-cycle regulators (Xie et al. 2010).

The bHLH-leucine zipper transcription factors ICE1/ SCREAM (SCRM) and SCRM2 are critical partners of SPCH, MUTE, and FAMA (Kanaoka et al. 2008). ICE1/ SCRM and SCRM2 proteins are expressed throughout the stomatal lineage, encompassing the expression patterns of SPCH, MUTE, and FAMA, and they can form physical associations with more restricted factors. ICE 1/ $S C R M$ and SCRM2 appear to have significant functional redundancy because both must be eliminated for a fully penetrant stomatal phenotype; in this case, the double mutant resembles spch (Kanaoka et al. 2008). A current 


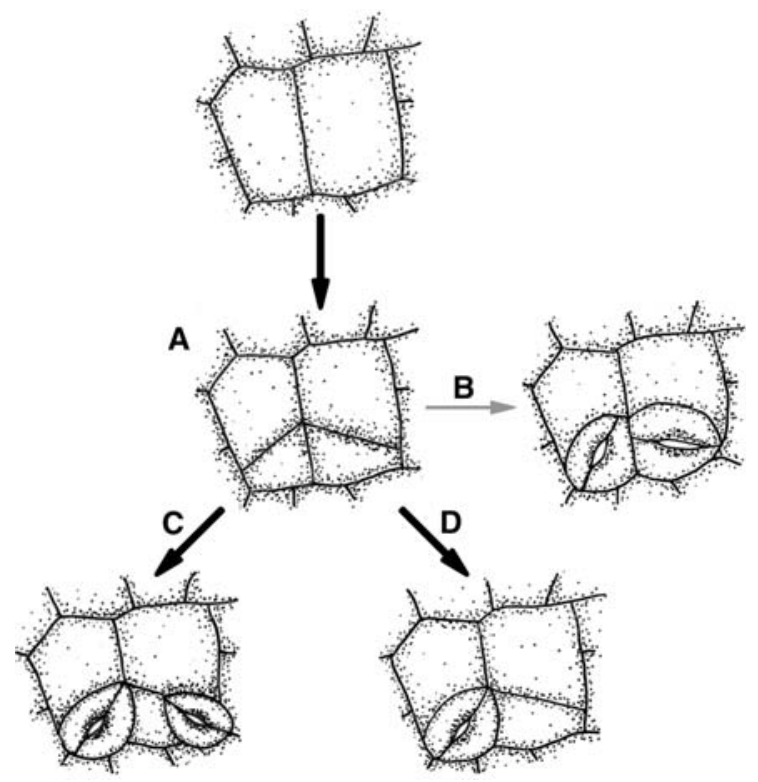

Figure 4. Error correction in the stomatal lineage. An intrinsic part of the developmental program of stomatal lineage cells is to correct errors. If two precursors are next to each other $(A)$, they rarely progress to both become stomata (an error; $B$ ) but instead fix the potential pattern error by undergoing additional cell divisions to create a "buffer" cell $(C)$ or undergoing arrest or transdifferentiation of a precursor $(D)$.

model is that a heterodimer between SPCH and ICE1/ SCRM or SCRM2 is required for priming protodermal cells for division and acquisition of meristemoid fate. MUTE and FAMA heterodimerization with ICE1/ SCRM2 would then be required for termination of asymmetric divisions and acquisition of GMC fate and for regulation of $\mathrm{GC}$ formation, respectively (Kanaoka et al. 2008). A single point mutation in ICE1/SCRM (SCRM-D) renders the protein overactive in promoting stomatal lineage fate, resulting in an epidermis composed entirely of stomata (Kanaoka et al. 2008). SCRM-D also reveals the mutual dependence of ICE1/SCRM and SPCH, MUTE, and FAMA because SCRM-D cannot overcome the requirement for any of the three genes to generate mature stomata (Kanaoka et al. 2008).

\section{Mechanisms to Establish Difference: Intrinsic Polarity}

The physically asymmetric divisions that characterize the stomatal lineage correlate with asymmetries in daughter cell behaviors and in a static image of a developing leaf; SPCH (and MUTE) often differentially mark the products of asymmetric divisions (MacAlister et al. 2007; Pillitteri et al. 2007). Time-lapse recording, however, indicates that SPCH is not segregated to one daughter cell but, rather, shows a distinct oscillating expression pattern in which expression peaks in a cell about to undergo an asymmetric division, is present in both daughters after division, and is then differentially extinguished in one daughter (Robinson et al. 2011). The continued presence of SPCH leads to continued rounds of asymmetric cell division, indicating a causal relationship between presence of the protein and stem-cell-like meristemoid behavior (Lampard et al. 2008; Robinson et al. 2011).

Asymmetry in SPCH expression as well as asymmetry in cell size impact meristemoid behavior. Mutations in BREAKING OF ASYMMETRY IN THE STOMATAL LIN$E A G E$ (BASL) lead to a prevalence of physically symmetric $\mathrm{MMC}$ cell divisions and an overproliferation and mispatterning of stomata (Dong et al. 2009). BASL, a novel protein found only in dicots, is asymmetrically localized at the cell cortex before cell divisions in the early stomatal lineage (Dong et al. 2009). Cortical BASL is inherited by the larger daughter cell after division, but, as with SPCH (and another polarized protein, POLAR [Pillitteri et al. 2011]), BASL shows differential behaviors in the daughters of asymmetric divisions. All three proteins are expressed (and re-expressed) during asymmetric divisions (BASL and POLAR in a polarized location) but all disappear in differentiating cells. Neither BASL nor POLAR is polarly localized in a SPCH mutant, suggesting that their activity is downstream from the specification of asymmetric division potential. One model for BASL and POLAR activity is that they reinforce distinct daughter cell fates by promoting physical differences between them (Dong et al. 2009; Pillitteri et al. 2011).

The behaviors of SPCH and BASL were predicted in the recent "polarity-switching" model for stomatal pattern. This model showed how a simple set of division rate and cell polarization rules could generate stomata surrounded by nonstomatal neighbors (Robinson et al. 2011). This is an elegant model for individual lineage behavior; however, it would require additional parameters to accommodate the interactions among multiple independent lineages (each a "point source" of stomatal production) simultaneously emerging next to one another and across the leaf (Fig. 5).

\section{Error Correction as a Pattern Mechanism}

In theory, correct stomatal pattern could arise from mechanisms that prevent stomatal precursors from being made in contact (prepattern) or through post hoc (errorcorrecting) mechanisms to deal with precursors that were made next to one another. Lineage tracing (Geisler et al. 2000) showed that meristemoids are not infrequently found next to other meristemoids, GMCs, or stomata. This inappropriate situation is resolved in one of two ways: Cells lose meristemoid identity and arrest or differentiate as a pavement cell, or, more frequently, the meristemoid divides again to create an SLGC "buffer" (Fig. 4). Either of these error-correcting mechanisms requires awareness of the neighbors.

The extent to which the stomatal lineage uses error correction rather than a prepattern may reflect a unique requirement of this developmental context. In contrast to organized regions of meristematic potential at the apices of the shoot and root that can be maintained throughout the life of the plant, stomatal lineages are transient and 
A
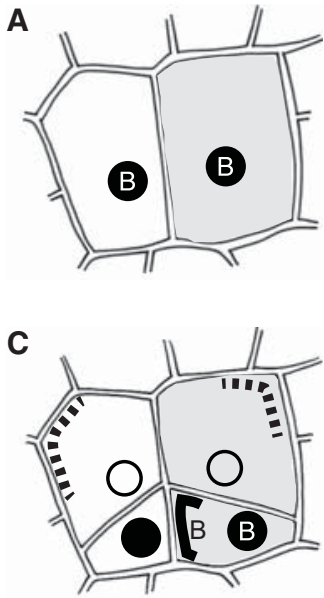

B

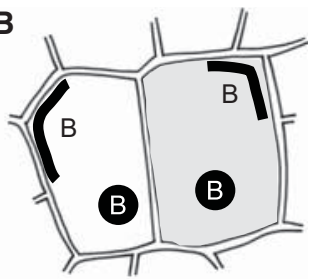

D

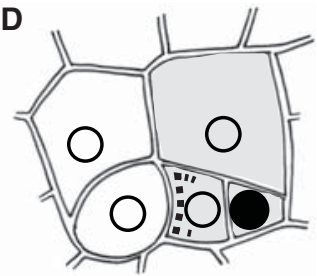

Figure 5. Extension of SPCH and BASL polarity model to multiple cells. Published models account for patterning among the progeny of a single cell lineage (Robinson et al. 2011). Often, however, independent lineages interact, as in this example of two initiating side by side. Gray shading distinguishes the lineages from one another. (A) MMCs become division competent accompanied by expression of SPCH (black nuclei) and nuclear BASL (circled "B"s). (B) BASL crescents form in the cell periphery, marking cell polarity (only one of several allowed axes of polarization is drawn for each cell). $(C, D)$ Asymmetric division of each MMC leads to small and large daughters, but their behavior is still flexible. In both lineages, the SLGC (upper and larger) cells are shown losing SPCH and BASL expression, a situation that corresponds to differentiation into pavement cells. In the left (white) lineage, the meristemoid (lower cell) inherits nuclear BASL and SPCH but fails to maintain them, a situation that leads to differentiation into a $\operatorname{GMC}(D)$. In the right (gray) stomatal lineage, however, SPCH and BASL are maintained and the meristemoid divides again, recapitulating the behavior of its mother. In this situation, however, the degrees of freedom for polarization axes are constrained and only the depicted division orientation is observed. Thus, pattern is not only a product of lineage-intrinsic polarity but is influenced by the neighboring lineage.

dispersed. Neither the orientation of stomata nor their precursors is aligned relative to each other or the leaf axis. In fact, the term "meristemoid" was originally coined to highlight cell populations whose growth trajectory was independent of body plan (Bünning 1953). This relative independence means that some of the common organizing principles and molecular mechanisms may not be available to the stomatal lineage, even when the cellular events are similar. For example, in both meristemoids and root stem cells, asymmetric divisions are critical components of cell fate and self-renewing capacity. In one example from the root, these divisions occur in a zone whose limits are defined by the integration of radial information (provided by movement of the transcription factor SHORTROOT from interior tissues) and longitudinal information determined by auxin distribution (Cruz-Ramirez et al. 2012). An important aspect of this system is that it can constrain asymmetric divisions to this specific location throughout the duration of root growth. Such a spatiotemporal positioning system is not well suited to the spatially unpredictable nature of stomatal initiation nor to the asynchronous timing of asymmetric div-

isions relative to organ development. Instead, what appears to be critical in stomatal development is the ability to distinguish one sister from another autonomously and to recognize immediate neighbors. The activity of asymmetrically distributed proteins such as BASL and POLAR may be the stomatal-specific solution to the first problem and peptide ligand/receptor-like kinase signaling (described below), a variation on a more general solution for the second.

Despite some differences, it is unlikely that stomatal development represents a completely new way of establishing developmental competence, pattern, and flexibility. A useful comparison then might be to look at other situations in which error correction takes place. One common situation in which there is rerouting of cell identity is in the context of injury. On removal of the root tip, differentiated (marker-displaying) cells can redefine their gene expression profiles and, over time, rebuild a morphologically normal root (Sena et al. 2009). When these experiments were performed in mutant backgrounds defective in building root organizers such as plethoral/plethora 2 or scarecrow, root tips were regenerated but could not grow indeterminately (Sena et al. 2009). These results were interpreted to mean that organogenesis and patterning can arise independent of clearly defined organizers, although indeterminate growth cannot - a situation with striking parallels to stomatal lineages.

\section{Mechanisms to Establish Difference: Signaling}

To solve the problems of recognizing neighbors and information provided by the environment, signaling is typically invoked. What might be the nature of these chemical or physical signals? On the basis of characterized mutants and cellular behavior, contact-mediated signals (and not diffusible signal gradients) are largely responsible. Four LRR receptors have been implicated in signal perception as plasma membrane ligand detectors: the receptor-like kinases ERECTA (ER), ERECTA-LIKE1 (ERL1), and ERECTA-LIKE2 (ERL2) and the receptor-like protein TOO MANY MOUTHS (TMM) (Nadeau and Sack 2002; Shpak et al. 2005; Lee et al. 2012). All four inhibit stomatal production in cotyledons and leaves, but $T M M$ shows a curious tissue-specific reversal of this role and is required to promote stomatal formation hypocotyls and stems (Geisler et al. 1998). These results suggest that TMM could modulate activity of the ERECTA family (ERf), dampening or enhancing signaling in a tissue-specific manner (Shpak et al. 2005; Abrash and Bergmann 2010).

The ligands for these receptors, all members of the EPIDERMAL-PATTERNING FACTOR LIKE (EPFL) family, are highly conserved in sequence and structure but vary in the output responses that they can generate and the cell types that synthesize them (Hara et al. 2007; Rychel et al. 2010; Abrash et al. 2011; Ohki et al. 2011). EPF1 and EPF2 are expressed in the stomatal lineage; EPF2 inhibits entry into the stomatal lineage and EPF1 acts later to prevent stomatal mispatterning (Hara et al. 2007, 2009; Hunt and Gray 2009). These stomatal re- 
pressing factors are counteracted by the activity of STOMAGEN (EPFL9), a stomatal promoting factor produced in interior tissues (Kondo et al. 2010; Sugano et al. 2010). Recent biochemical studies showed that ER and ERL1 receptor kinases form homo- or hetero-oligomers between each other or with TMM but that TMM cannot interact with itself (Lee et al. 2012). ER, ERL1, and TMM also have different binding kinetics and affinities to synthetic versions of the ligands EPF1 and EPF2 (Lee et al. 2012). These data, in conjunction with distinctive expression patterns for the receptors, are consistent with models suggesting that receptor complexes undergo dynamic shuffling of members leading to a temporal succession of differential signaling capabilities during stomatal development (see detailed models in Lee and Torii, this volume).

\section{Intracellular Signal Transduction: The Complex Case of MAPK Signaling}

Connecting the activities at the cell periphery with the complex transcriptional and morphological responses are members of MAPK cascades. In Arabidopsis, this threetier cascade consists of $20 \mathrm{MAPKs}$ (MPKs) that are the targets of 10 upstream MAPKKs (MKKs), themselves targets for more than 80 MAPKKKs. The MAPK cascade was first recognized as negatively regulating stomatal differentiation when mutants for the MAPKKK YODA were isolated (Bergmann et al. 2004). Subsequent studies found MKK4/5/7/9 and MPK3/6 as downstream members of the cascade (Wang et al. 2007; Lampard et al. 2009). Of these MAPK members, MPK3/6 and MKK4/ 5 were well known to mediate stress and pathogen responses so it is somewhat puzzling as to why, given the large number of MAPKs available, the same few members are used in so many developmental and homeostatic situations. There is, however, some evidence for specificity among MAPK cascade members; when all 10 MKKs were assayed, only four affected stomatal development (Lampard et al. 2009; GR Lampard, unpubl.).

\section{THE USE OF STOMATAL LINEAGE AS A TOOL TO DISSECT MAPK SIGNALING}

Using overlapping signaling and transcriptional networks, such as the MAPKs and their downstream effectors, requires strategies to maximize useful information but minimize interfering cross talk. How does the stomatal lineage solve such a problem? How can the lineage be exploited to find general principles of signaling specificity?

Stomatal lineage cells independently and asynchronously arise in close proximity to one another and to other nonstomatal lineage cells. This scenario suggests that a robust cell-cell dialogue must be used to generate pattern and promote optimal distributions for a particular environmental situation. As described above, signaling from multiple ligands and receptors converges on a common MAPK cascade whose output determines cell fates. Further complicating this are contemporaneous environmental inputs that could modulate outcomes upstream of, downstream from, or even within the MAPK pathway (see, e.g., Kim et al. 2012). How do cells, with so many signals being funneled into (potentially) the same transduction pathway, make a decision? Although a very complex signaling context, the output here is relatively simple. Stomatal lineage cells have a restricted number of cell-fate options: divide, arrest, or differentiate. The streamlining of complex signal input to simple output might reside in the MAPK cascade, in accessories to the core cascade, or in targets downstream.

\section{SPCH as a MAPK Sensor}

Integrative decision-making sensors readjust cellular outputs to the immediate conditions, provided by the neighboring cells and the environment. In mammals, a subset of MAPKs called ERKs are differentially activated to provide fate-switch control (Murphy and Blenis 2006). In fibroblasts, sustained but not transient ERK activity activates the cell cycle. In rat adrenal medulla cells, in contrast, it is transient ERK activation that induces a proliferative burst, and sustained activation leads to differentiation into neurons (Murphy et al. 2002; Murphy and Blenis 2006). Therefore, there are both cell-type-specific and signal-input-specific ways to modulate MAPK signaling. But what is the MAPK signal-duration sensing mechanism? In fibroblasts, one mechanism underlying differential responses to ERK activity duration is the behavior of the transcription factor c-Fos (Murphy et al. 2002). The c-Fos gene belongs to a group of immediateearly genes whose expression is up-regulated upon transient or sustained MAPK activation. c-Fos is a highly labile protein and requires phosphorylation for stabilization. Activation of transcriptional regulation competence depends on further phosphorylation of additional sites. Because of this, during transient ERK activation, there is a temporal separation between active ERK and c-Fos protein; thus, unphosphorylated c-Fos is degraded. Under conditions of sustained ERK activation, c-Fos is phosphorylated, stabilized, and activated, resulting in CycD1 expression and fibroblast proliferation (Murphy et al. 2002). Using this strategy, mammalian cells are capable of determining the duration of a MAPK signal and responding appropriately by activating a transcriptional program.

Are there analogous molecular elements that, like ERK and c-Fos, could act as MAPK sensors in stomatal differentiation? One strong candidate is SPCH. SPCH has multiple canonical and noncanonical MAPK-target sites that have been shown to be in vitro MAPK targets and in vivo negative regulators of SPCH activity (Lampard et al. 2008; Gudesblat et al. 2012). Although opposite in direction to MAPK/c-Fos, MAPK-mediated phosphorylation and destabilization of SPCH could also act as a cell-fate switch in plants.

A SPCH-based sensing mechanism could be active during (1) the selection of MMCs from protodermal cells and their subsequent asymmetric cell division, (2) meristemoid amplifying divisions, and (3) the generation of satellite meristemoids by the divisions of SLGCs. U1- 
timately, these three situations are similar, each resulting in asymmetric divisions and production of meristemoids. In history, however, these cells differ, with the meristemoid and SLGC having previously expressed SPCH but only the former of these continuously. It is not known whether these three SPCH-dependent decisions would use identical regulatory mechanisms. Regardless of how similar or different stomatal lineage cells are, their endogenous MAPK signaling is likely to be nuanced and could include activation as well as inhibition of SPCH at a particular developmental step. Quantitatively different division-promoting behavior of SPCH was seen with successive elimination of MAPK target sites (Lampard et al. 2008), and a qualitative shift in fate-promoting behavior occurs when five or more sites are eliminated (Lampard et al. 2008). These observations suggest how this sensing mechanism might work. MAPK signaling output would be a function of the SPCH phosphorylation state, with hypophosphorylation correlating with increased asymmetric cell divisions and hyperphosphorylation with the propensity to promote differentiation as pavement cells (Lampard et al. 2008, 2009). Expression of CA-MKKs under the SPCH promoter generates a phenotype identical to loss of SPCH (Lampard et al. 2009), suggesting that hyperphosphorylation correlates with constitutive degradation of SPCH (Lampard et al. 2008).

We do not know whether phosphorylation status of SPCH primarily regulates stability such that differences relate to the length of time in which SPCH can engage transcriptional targets or whether phosphorylation also creates a differentially active SPCH. It is conceivable, for example, that some phospho-states promote SPCH interactions with ICE1/SCRM and SCRM2 or that a specific state might bias interaction toward one or the other of these partners. Moreover, the three cellular contexts in which SPCH acts as a sensor may differ in their expression of other SPCH partners or, owing to previous SPCH expression, may be sensitized or buffered against additional SPCH activity.

Continuing the analogy from mammalian systems, SPCH activity (both amount and type) in these different stages could depend on the strength and duration of the MAPK signal. What then modulates plant MAPK signaling profiles? Input strength or duration could vary; for example, EPFL family peptide signals acting through TMM and ERf receptors could provide a relatively longlived "developmental" signal, whereas stress or pathogens might induce a short-lived but strong signal. The source of these inputs could also vary. Developmental signals could originate from different tissues and have opposite effects. STOMAGEN is expressed in the mesophyll during an extended period of leaf development (Hunt et al. 2010; Kondo et al. 2010; Sugano et al. 2010). EPF1 and EPF2, in contrast, are expressed in discrete "point source" cells in the epidermis and might contribute more locally to cell behavior and pattern. Stress signals could originate from individual leaf cells or reflect a systemic response and could interface with SPCH regulation through activation of MPK3/6 activity. An interesting test case for signal integration would be in meristemoids of water-limited plants. Recent work from Skirycz et al. (2011) showed that when subjected to transient mild drought, meristemoids pause divisions but can resume them when re-watered. Activation of MAPK signaling in meristemoids would be expected to lead to SPCH hyperphosphorylation and degradation, whereas the resumption of division would require new SPCH synthesis. If SPCH "sensing" is key to this environmental response, we would predict that meristemoid divisions in SPCH phospho-site mutants would not arrest in drought conditions. Should this be that case, the system would be useful to dissect the level at which integration lies. In drought situations, EPFL-mediated signaling could synergize (in the case of stomatal repressors EPF1/EPF2) or antagonize (STOMAGEN) stress signaling at the level of YODA, MKK, or MAPK activation. Alternatively, drought signaling could be mediated through the modulation of EPFL abundance or activity, indicating that the MAPK cascade serves as a downstream effector of a decision made through signal integration at the cell surface.

\section{Other Fate Transition Sensors in the Stomatal Lineage}

In addition to SPCH-based transitions, other important developmental decisions must be made in the stomatal lineage. When late meristemoids express constitutively active versions of MKK4 (or 5, 7, or 9) stomatal differentiation arrests before GMC formation (Lampard et al. 2009). SPCH, however, is not expressed at this developmental stage. What serves as later MAPK activity sensor is not known, but promising candidates include other stomatal transcription factors MUTE, ICE1, and MYB88, shown by a protein microarray-based library screen (Popescu et al. 2009) or in other biochemical assays (Miura et al. 2011) to be substrates of MAPK phosphorylation. Whether these transcription factors can indeed interpret MAPK-mediated phosphorylation into differential cell fate outcomes is yet to be determined.

\section{MKKs in the GMC-to-GC Transition Are an Experimental Framework to Unlock MAPK Specificity}

Activation of MAPK signaling in MMCs or meristemoids either arrests these precursors or forces them out of the stomatal lineage. Surprisingly, during the final stage of stomatal development (the GMC-to-GC transition), the direction of the MAPK effect switches, promoting rather than repressing stomatal development. In a phenotype reminiscent of c-Fos activation by sustained ERK signaling, CA-MKK7/9 expression results in proliferation of GMCs and development of stomatal clusters (Lampard et al. 2009). This is a unique phenotype that produces numerous "complete" stomata (composed of two sister guard cells) piled on top of one another at the site of the original GMC. Interestingly, at this stage, CAMKK4/5 are no longer capable of altering stomatal development and only CA-MKK7/9 have an effect. Sto- 
mata in the CA-MKK7/9-induced clusters are arranged randomly with respect to one another. This phenotype contrasts with the "side-by-side" stomata pheno-types associated with loss of FAMA or FLP;MYB88 (Lai et al. 2005; Ohashi-Ito and Bergmann 2006) and suggests that the MAPK-mediated reprogramming occurs before the establishment of GMC polarity. The differences in phenotypes also rule out FAMA and FLP/MYB88 as the sole downstream MAPK effectors and imply that immediately after acquiring GMC identity, an unknown MAPK-responsive factor might also act as a sensor to translate MKK7/9-dependent signaling into proliferation and differentiation.

Different MAPK network topologies (elements and their connections) can lead to different phenotypes. This is seen, for example, in the unique ability of CAMKK7/9 to promote proliferation in the GMC-to-GC transition and the ability of CA-MKK4/5 (but not CAMKK7/9) to rescue the reduced cell divisions and short pedicel phenotype of erecta (Meng et al. 2012). This poses a dilemma, however, when considering the earlier stomatal lineage stages in which multiple MAPK topologies are permitted. CA-MKK4/5 and CA-MKK7/9 are equally capable of inhibiting stomatal formation. Either some cells purposely overlook or discard information about MAPK topologies during signal-output processing or some cells acquire a new ability to discriminate among MAPK topologies. Currently, we lack data to distinguish among these hypotheses, but they do inspire some questions. Might MMCs and meristemoids use the same MAPK network topology because they are both normally "transient" cell types? Does this transient property explain why the output of MAPK signaling is inhibition of cell division? What is the critical difference at the GMC stage? A future frontier in plant development will be to understand how cells assemble specific MAPK networks and "read" these topologies to generate a consistent and appropriate response. Essentially, the task is to build the wiring diagrams behind logic decisions and link them to the generation of specific phenotypes.

To link cellular logic to phenotype generation, networks need to be simplified and explored to isolate network nodes and their connections. In this regard, the emergent specificity in the GMC-to-GC transition is particularly suitable to study MAPK topology "interpreters." Progressive dissection of MKK7/9 proteins can be performed to isolate intrinsic motifs that distinguish them from MKK4/5. Recent results from domain swap experiments in our laboratory (DL Wengier, unpubl.) suggest that MKK5 and MKK7 proteins possess regions outside of the conserved kinase domain that account for differential behaviors of these MKKs. These motifs act in a modular fashion and can change MKK identity when swapped between MKK5 and MKK7. The motifs and the phenotypes that MKKs containing them confer can also be used in biochemical and genetic interaction screens to identify extrinsic factors than confer specificity. The MKK extrinsic factors may be downstream MPKs with different affinities for the MKKs or MPKs that are present in only some cell types. Alternatively, these motifs might attract the elusive plant MAPK scaffold proteins that, like their yeast or mammalian counterparts, assemble specific kinase cascades and modulate the activity of those cascades (enhancing or dampening them) especially in situations in which a cell must integrate environmental cues into its developmental program.

\section{Cross Talk in the Stomatal MAPK Pathway: A Spatial Solution?}

Reinforcing the importance of scaffolds and coordinating MAPKs with multiple factors is recent work showing that the MAPK pathway also intersects with hormone signaling. Kim et al. (2012) showed that the brassinosteroid response kinase BIN2 phosphorylated and negatively regulated the MAPKKK YODA, alleviating the inhibition that the MAPK pathway confers on SPCH and stomatal initiation. Application of bikinin (a BIN2 inhibitor) activated MAPK pathway activity and repressed stomatal development (Kim et al. 2012). A complication in the story is that SPCH itself appears to be a direct target of BIN2. Gudesblat et al. (2012) showed that BIN2 can phosphorylate a $\mathrm{SPCH}$ amino-terminal region, leading to a destabilization of SPCH, potentially independent of the regulation of SPCH by MAPK-mediated phosphorylation. In hypocotyl assays, inactivation of BIN2 correlated with an increase in stomatal production (Gudesblat et al. 2012).

The Kim and Gudesblat results seem to conflict in terms of the role of BR signaling in stomatal development and in how this signaling interfaces with YODA and $\mathrm{SPCH}$. In a trivial sense, technical differences in growth conditions and the analysis of different tissues by the two groups could lead to the impression of contradiction. It has already been shown that mutations in TMM result in opposite effects on stomatal production in hypocotyls and cotyledons (Geisler et al. 1998); thus, opposing phenotypes in these two organs may be consistent with disruption of a common process. More interesting, however, is the idea that BR signaling, as mediated through the activity of BIN2, really does engage in biochemical cross talk at multiple levels of the stomatal development pathway. What might be the logic in this? Theoretical models of signal transduction often include opposing modulation of downstream effectors to permit high sensitivity, finetuning, or robustness in a decision (Brandman and Meyer 2008). These explanations assume that both positive and negative effects occur in the same cell. An alternative (although not mutually exclusive) hypothesis is that BR exerts differential effects in a cell-type-specific manner (Fig. 6). In meristemoids, BR-mediated inhibition of BIN2 could inhibit BIN2-dependent degradation of $\mathrm{SPCH}$, allowing these cells to maintain their self-renewing capability. In SLGCs, the well-established role of BR in cell elongation could come into play; here, inhibition of BIN2 would allow activation of the MAPK cascade and thus down-regulation of SPCH. This would serve to promote exit from the self-renewing (SPCH-expressing) state, while promoting the cell expansion typical of SLGCs. 


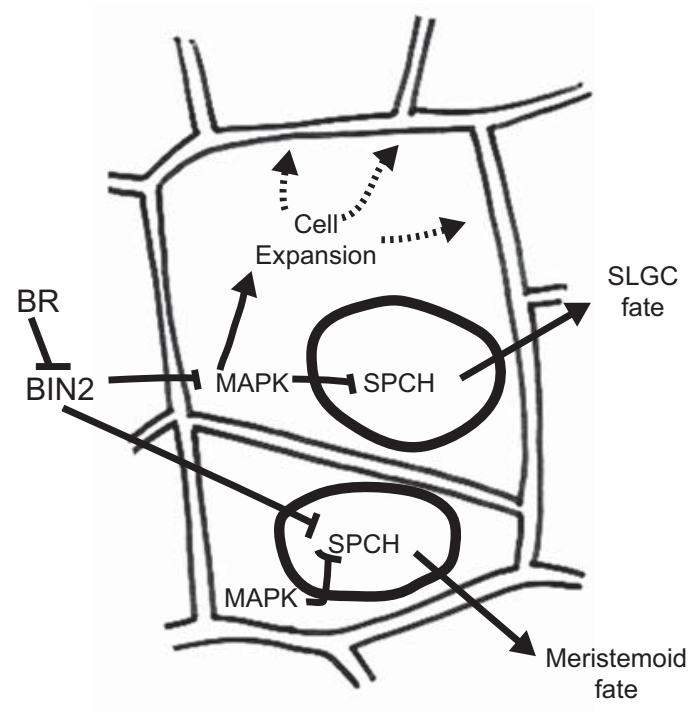

Figure 6. A model for spatial specificity of hormone signaling in stomatal lineage cells. Recent studies have come to conflicting conclusions about the roles of brassinosteroid (BR) hormone in stomatal identity and in regulation of the transcription factor SPCH. A hypothetical (but testable) resolution for this conundrum is to consider spatial separation of signaling responses: In meristemoids, BR would inactivate BIN2-dependent degradation of SPCH, committing these cells to their fate; in SLGCs, BR would release BIN2 from inhibiting the MAPK cascade, thus resulting in phosphorylation and degradation of SPCH (and promoting exit from stomatal lineage fate). Other effectors of MAPK or BR would simultaneously promote cell expansion in this cell.

Future studies focused on individual cell responses made possible by genetic mosaic experiments such as those performed with expression of the BR receptor in the epidermis (Savaldi-Goldstein et al. 2007) or by the creation of spatially resolved sensors of BR activity (akin to those for other plant hormones) (Muller and Sheen 2008; Brunoud et al. 2012) could resolve these issues.

\section{CONCLUSIONS}

Plant cells undergo a series of decision-making processes during their lifetimes. Analysis of the logical structures underpinning those decisions and the physical elements that can be wired together to manifest such structures is still in its infancy. The stomatal lineage possesses major developmental features of asymmetric and oriented cell divisions, stem-cell-like behaviors, cellcell communication, and environmental sensitivity. Yet, at the same time, the cellular outcomes of complex inputs are easily monitored and discrete. Together, these properties make the system useful for network discovery and synthetic biology approaches. Importantly, candidate signaling pathways (MAPKs) and transcriptional regulators (SPCH) that could serve as major integration hubs have already been identified. The particulars of MAPK cascade assemblages and behaviors during different cell-state- transition stages provide a unique opportunity to uncover signaling topology interpreters as well as potentially enabling the synthetic approach of "rewiring" signaling input/output relationships. On the basis of the ability of plants to recruit preexisting pathways for specific decisions, we envision that studies of the stomatal development program will lead to discovery of new sets of gene families, mechanisms, and developmental "rules" that shape plants and enable them to flourish in the world that surrounds them.

\section{ACKNOWLEDGMENTS}

We are indebted to the classical embryologists and mathematicians who formulated interesting questions decades ago and to laboratory members and colleagues in the developmental biology community for inspiring attempts to answer these questions. D.C.B is a Gordon and Betty Moore Foundation investigator of the Howard Hughes Medical Institute.

\section{REFERENCES}

Abrash EB, Bergmann DC. 2010. Regional specification of stomatal production by the putative ligand CHALLAH. Development 137: 447-455.

Abrash EB, Davies KA, Bergmann DC. 2011. Generation of signaling specificity in Arabidopsis by spatially restricted buffering of ligand-receptor interactions. Plant Cell 23: 2864-2879.

Berger F, Linstead P, Dolan L, Haseloff J. 1998. Stomata patterning on the hypocotyl of Arabidopsis thaliana is controlled by genes involved in the control of root epidermis patterning. Dev Biol 194: 226-234.

Bergmann DC, Lukowitz W, Somerville CR. 2004. Stomatal development and pattern controlled by a MAPKK kinase. Science 304: 1494-1497.

Bharathan G, Goliber TE, Moore C, Kessler S, Pham T, Sinha NR. 2002. Homologies in leaf form inferred from KNOXI gene expression during development. Science 296: 1858-1860.

Brandman O, Meyer T. 2008. Feedback loops shape cellular signals in space and time. Science 322: 390-395.

Brunoud G, Wells DM, Oliva M, Larrieu A, Mirabet V, Burrow AH, Beeckman T, Kepinski S, Traas J, Bennett MJ, et al. 2012. A novel sensor to map auxin response and distribution at high spatio-temporal resolution. Nature 482: 103-106.

Bünning E. 1953. Entwicklungs- und Bewegungsphysiologie der Pflanze. Springer-Verlag, Berlin.

Casson S, Gray JE. 2008. Influence of environmental factors on stomatal development. New Phytol 178: 9-23.

Cruz-Ramirez A, Diaz-Trivino S, Blilou I, Grieneisen VA, Sozzani R, Zamioudis C, Miskolczi P, Nieuwland J, Benjamins R, Dhonukshe P, et al. 2012. A bistable circuit involving SCARECROW-RETINOBLASTOMA integrates cues to inform asymmetric stem cell division. Cell 150: 1002-1015.

Dong J, MacAlister CA, Bergmann DC. 2009. BASL controls asymmetric cell division in Arabidopsis. Cell 137: 13201330 .

Geisler M, Yang M, Sack FD. 1998. Divergent regulation of stomatal initiation and patterning in organ and suborgan regions of the Arabidopsis mutants too many mouths and four lips. Planta 205: 522-530.

Geisler M, Nadeau J, Sack FD. 2000. Oriented asymmetric divisions that generate the stomatal spacing pattern in Arabidopsis are disrupted by the too many mouths mutation. Plant Cell 12: 2075-2086.

Gudesblat GE, Schneider-Pizon J, Betti C, Mayerhofer J, Vanhoutte I, van Dongen W, Boeren S, Zhiponova M, de Vries S, 
Jonak C, et al. 2012. SPEECHLESS integrates brassinosteroid and stomata signalling pathways. Nat Cell Biol 14: 548-554.

Hara K, Kajita R, Torii KU, Bergmann DC, Kakimoto T. 2007. The secretory peptide gene EPF1 enforces the stomatal onecell-spacing rule. Genes Dev 21: 1720-1725.

Hara K, Yokoo T, Kajita R, Onishi T, Yahata S, Peterson KM, Torii KU, Kakimoto T. 2009. Epidermal cell density is autoregulated via a secretory peptide, EPIDERMAL PATTERNING FACTOR 2 in Arabidopsis leaves. Plant Cell Physiol 50: 1019-1031.

Hunt L, Gray JE. 2009. The signaling peptide EPF2 controls asymmetric cell divisions during stomatal development. Curr Biol 19: 864-869.

Hunt L, Bailey KJ, Gray JE. 2010. The signalling peptide EPFL9 is a positive regulator of stomatal development. New Phytol 186: 609-614.

Kanaoka MM, Pillitteri LJ, Fujii H, Yoshida Y, Bogenschutz NL, Takabayashi J, Zhu J-K, Torii KU. 2008. SCREAM/ ICE1 and SCREAM2 specify three cell-state transitional steps leading to Arabidopsis stomatal differentiation. Plant Cell 20: $1775-1785$.

Kim TW, Michniewicz M, Bergmann DC, Wang ZY. 2012. Brassinosteroid regulates stomatal development by GSK3-mediated inhibition of a MAPK pathway. Nature 482: 419-422.

Kondo T, Kajita R, Miyazaki A, Hokoyama M, NakamuraMiura T, Mizuno S, Masuda Y, Irie K, Tanaka Y, Takada S, et al. 2010. Stomatal density is controlled by a mesophyllderived signaling molecule. Plant Cell Physiol 51: 1-8.

Lai LB, Nadeau JA, Lucas J, Lee E-K, Nakagawa T, Zhao L, Geisler M, Sack FD. 2005. The Arabidopsis R2R3 MYB proteins FOUR LIPS and MYB88 restrict divisions late in the stomatal cell lineage. Plant Cell 17: 2754-2767.

Lampard GR, MacAlister CA, Bergmann DC. 2008. Arabidopsis stomatal initiation is controlled by MAPK-mediated regulation of the bHLH SPEECHLESS. Science 322: 1113-1116.

Lampard GR, Lukowitz W, Ellis BE, Bergmann DC. 2009. Novel and expanded roles for MAPK signaling in Arabidopsis stomatal cell fate revealed by cell type-specific manipulations. Plant Cell 21: 3506-3517.

Lee JS, Kuroha T, Hnilova M, Khatayevich D, Kanaoka MM, McAbee JM, Sarikaya M, Tamerler C, Torii KU. 2012. Direct interaction of ligand-receptor pairs specifying stomatal patterning. Genes Dev 26: 126-136.

MacAlister CA, Ohashi-Ito K, Bergmann DC. 2007. Transcription factor control of asymmetric cell divisions that establish the stomatal lineage. Nature 445: 537-540.

Meng X, Wang H, He Y, Liu Y, Walker JC, Torii KU, Zhang S. 2012. A MAPK cascade downstream of ERECTA receptorlike protein kinase regulates Arabidopsis inflorescence architecture by promoting localized cell proliferation. Plant Cell 12: $4948-4960$.

Miura K, Ohta M, Nakazawa M, Ono M, Hasegawa PM. 2011. ICE1 Ser403 is necessary for protein stabilization and regulation of cold signaling and tolerance. Plant J 67: 269-279.

Muller B, Sheen J. 2008. Cytokinin and auxin interaction in root stem-cell specification during early embryogenesis. Nature 453: 1094-1097.
Murphy LO, Blenis J. 2006. MAPK signal specificity: The right place at the right time. Trends Biochem Sci 31: 268275.

Murphy LO, Smith S, Chen RH, Fingar DC, Blenis J. 2002. Molecular interpretation of ERK signal duration by immediate early gene products. Nat Cell Biol 4: 556-564.

Nadeau JA, Sack FD. 2002. Control of stomatal distribution on the Arabidopsis leaf surface. Science 296: 1697-1700.

Ohashi-Ito K, Bergmann DC. 2006. Arabidopsis FAMA controls the final proliferation/differentiation switch during stomatal development. Plant Cell 18: 2493-2505.

Ohki S, Takeuchi M, Mori M. 2011. The NMR structure of stomagen reveals the basis of stomatal density regulation by plant peptide hormones. Nat Commun 2: 512.

Pillitteri LJ, Sloan DB, Bogenschutz NL, Torii KU. 2007. Termination of asymmetric cell division and differentiation of stomata. Nature 445: 501-505.

Pillitteri LJ, Peterson KM, Horst RJ, Torii KU. 2011. Molecular profiling of stomatal meristemoids reveals new component of asymmetric cell division and commonalities among stem cell populations in Arabidopsis. Plant Cell 23: 3260-3275.

Popescu SC, Popescu GV, Bachan S, Zhang Z, Gerstein M, Snyder M, Dinesh-Kumar SP. 2009. MAPK target networks in Arabidopsis thaliana revealed using functional protein microarrays. Genes Dev 23: 80-92.

Robinson S, Barbier de Reuille P, Chan J, Bergmann D, Prusinkiewicz P, Coen E. 2011. Generation of spatial patterns through cell polarity switching. Science 333: 1436-1440.

Rychel AL, Peterson KM, Torii KU. 2010. Plant twitter: Ligands under 140 amino acids enforcing stomatal patterning. J Plant Res 123: 275-280.

Savaldi-Goldstein S, Peto C, Chory J. 2007. The epidermis both drives and restricts plant shoot growth. Nature 446: 199-202.

Sena G, Wang X, Liu HY, Hofhuis H, Birnbaum KD. 2009. Organ regeneration does not require a functional stem cell niche in plants. Nature 457: 1150-1153.

Shpak ED, McAbee JM, Pillitteri LJ, Torii KU. 2005. Stomatal patterning and differentiation by synergistic interactions of receptor kinases. Science 309: 290-293.

Skirycz A, Claeys H, De Bodt S, Oikawa A, Shinoda S, Andriankaja M, Maleux K, Eloy NB, Coppens F, Yoo S-D, et al. 2011. Pause-and-stop: The effects of osmotic stress on cell proliferation during early leaf development in Arabidopsis and a role for ethylene signaling in cell cycle arrest. Plant Cell 23: 1876-1888.

Sugano SS, Shimada T, Imai Y, Okawa K, Tamai A, Mori M, Hara-Nishimura I. 2010. Stomagen positively regulates stomatal density in Arabidopsis. Nature 463: 241-244.

Wang H, Ngwenyama N, Liu Y, Walker JC, Zhang S. 2007. Stomatal development and patterning are regulated by environmentally responsive mitogen-activated protein kinases in Arabidopsis. Plant Cell 19: 63-73.

Xie Z, Lee E, Lucas JR, Morohashi K, Li D, Murray JAH, Sack FD, Grotewold E. 2010. Regulation of cell proliferation in the stomatal lineage by the Arabidopsis MYB FOUR LIPS via direct targeting of core cell cycle genes. Plant Cell 22: 23062321. 


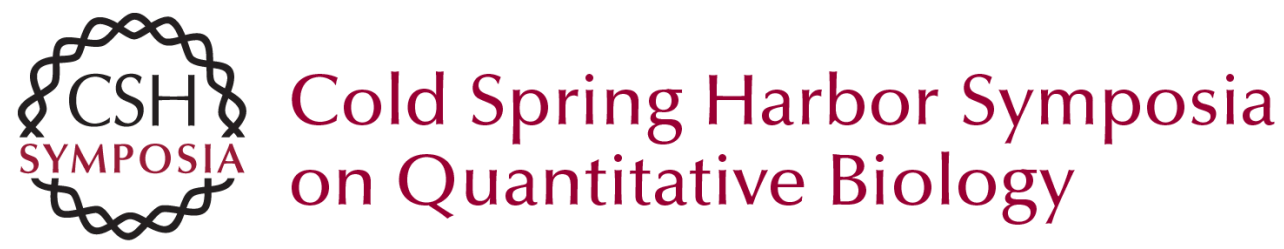

\title{
On Fate and Flexibility in Stomatal Development
}

\author{
D.L. Wengier and D.C. Bergmann
}

Cold Spring Harb Symp Quant Biol 2012 77: 53-62 originally published online February 26, 2013 Access the most recent version at doi:10.1101/sqb.2013.77.015883

References This article cites 45 articles, 21 of which can be accessed free at: http://symposium.cshlp.org/content/77/53.full.html\#ref-list-1

\section{License}

Email Alerting Receive free email alerts when new articles cite this article - sign up in Service the box at the top right corner of the article or click here. 